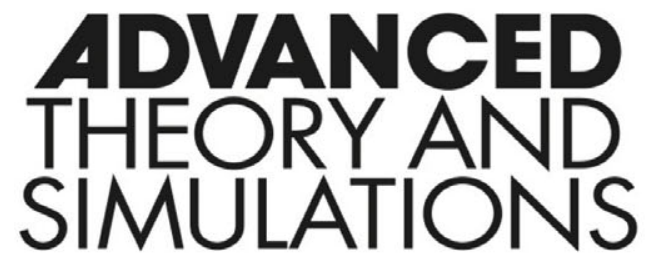

Postfach 101161 69451 Weinheim

Germany WILEY-VCH

Courier services:

Boschstraße 12

69469 Weinheim

Germany

Tel.: (+49) 6201606179

Fax: (+49) 6201606500

E-mail: advtheorysimul@wiley.com

Dear Author,

Please correct your galley proofs carefully and return them no more than four days after the page proofs have been received.

Please limit corrections to errors already in the text; cost incurred for any further changes or additions will be charged to the author, unless such changes have been agreed upon by the editor.

The editors reserve the right to publish your article without your corrections if the proofs do not arrive in time.

Note that the author is liable for damages arising from incorrect statements, including misprints.

Please note any queries that require your attention. These are indicated with a $Q$ in the PDF and a question at the end of the document.

Reprints may be ordered by filling out the accompanying form.

Return the reprint order form by fax or by e-mail with the corrected proofs, to WileyVCH : advtheorysimul@wiley.com
To avoid commonly occurring errors, please ensure that the following important items are correct in your proofs (please note that once your article is published online, no further corrections can be made):

- Names of all authors present and spelled correctly

- Titles of authors correct (Prof. or Dr. only: please note, Prof. Dr. is not used in the journals)

- Addresses and postcodes correct

- E-mail address of corresponding author correct (current email address)

- Funding bodies included and grant numbers accurate

- Title of article OK

- All figures included

- Equations correct (symbols and sub/superscripts)

Corrections should be made directly in the PDF file using the PDF annotation tools. If you have questions about this, please contact the editorial office. The corrected PDF and any accompanying files should be uploaded to the journal's Editorial Manager site. 


\section{Author Query Form}

Journal ADTS

Article adts201800203

Dear Author,

During the copyediting of your manuscript the following queries arose.

Please refer to the query reference callout numbers in the page proofs and respond to each by marking the necessary comments using the PDF annotation tools.

Please remember illegible or unclear comments and corrections may delay publication.

Many thanks for your assistance.

\begin{tabular}{|c|c|c|}
\hline Query No. & Description & Remarks \\
\hline Q1 & Please provide a TOC keyword that is suitable for this paper. & Membraneless organelles \\
\hline Q2 & $\begin{array}{l}\text { Please confirm that forenames/given names (blue) and surnames/family names (vermil- } \\
\text { ion) have been identified correctly. }\end{array}$ & Yes \\
\hline Q3 & $\begin{array}{l}\text { Please define all acronyms at their first appearance in the abstract, text and table of con- } \\
\text { tents, respectively. Only expanded forms are allowed if the elements are cited only once in } \\
\text { the article. }\end{array}$ & $\begin{array}{l}\text { SH3 = SRC Homolgy domain } \\
3 .\end{array}$ \\
\hline Q4 & $\begin{array}{l}\text { Please provide the highest academic title (either Dr. or Prof.) for all authors, where appli- } \\
\text { cable. }\end{array}$ & $\begin{array}{l}\text { Dr Martin Sweatman } \\
\text { Prof. Robert Insall }\end{array}$ \\
\hline Q5 & Please check all equations have been correctly typeset. & Yes \\
\hline Q6 & Please provide keywords (maximum of 5, plural, all abbreviations defined). & * \\
\hline Q7 & Please update ref. [7]. & doi: 10.1074/ \\
\hline
\end{tabular}

* membraneless organelles, molecular simulation, actin nucleation, self-limited aggregation, competing interactions 


\section{FULL PAPERS}

Q1

\section{$x x x$}

M. B. Sweatman,* R. Insall . . 1800203

Assembly of the Actin Catalyst WASP

by Giant SALR Cluster Formation

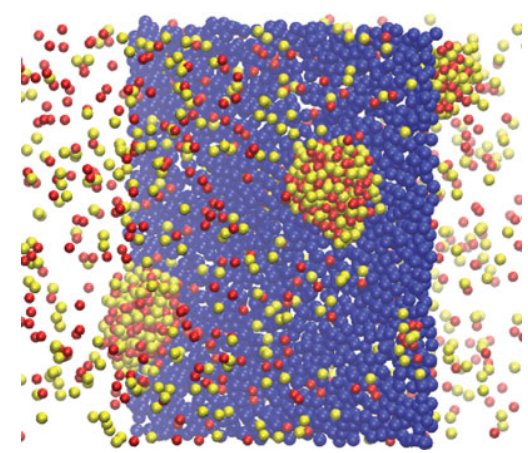

Membraneless organelles in biological cells are generally thought to form via liquid-liquid phase separation. However, using Monte Carlo simulation, it is shown that the SALR (short-range attraction, long-range repulsion) interaction model provides a better description of the physics of one kind of membraneless organelle, known as WASP puncta, at a model cell membrane. 


\title{
Assembly of the Actin Catalyst WASP by Giant SALR Cluster Formation
}

\author{
Martin B. Sweatman* and Robert Insall
}

Some structures in living cells behave like organelles, but lack individual membranes. The mechanisms that produce such "membraneless organelles" are not understood, but are commonly thought to involve the formation of separate phases via liquid-liquid phase separation. Here, an alternative mechanism is investigated involving short-range attraction, long-range repulsion (SALR) fluids, applied at model biological membrane-cytoplasm interfaces. Using Monte Carlo simulations, it is found that giant SALR clusters can form at the membrane surface even when they are unstable in the bulk, for suitably chosen model parameters. It is also found that the formation and location of giant SALR clusters at the interface can be controlled by a third protein species confined to the membrane surface. This behavior mimics that of WASP puncta at the cell membrane, thought to involve WASP and Nck protein aggregates controlled by clathrin. The SALR mechanism is thus a good model for this system, and for membraneless organelles more generally.
In a typical cell, the Wiskott Aldrich Syndrome protein (WASP) and its relatives (the WASP family) account for the majority of nucleation, and therefore most of the actin assembled at any time. ${ }^{[2]}$ However, their localization near the cell membrane is not understood. One interesting feature is their discreteness. WASP itself is normally found only in small puncta, or dimples, in the cell membrane. Its relative, the SCAR/WAVE protein, is normally found in dynamic streaks just inside the protruding edge of moving cells. ${ }^{[3]}$

The principal biological role of the actin polymer produced by WASP puncta involves endocytosis, the mechanism through which cells internalize cell membrane. During this process, small pits in the membrane form, lined by the protein clathrin. Clathrin-coated pits typically remain on the

\section{Introduction}

Cells move by polymerizing actin, which is a small and simple protein present in high concentrations within the cell. One key feature of actin polymerization is that it is self-driven-no other components are needed to drive filament extension. However, cells require that actin polymerization be focused at particular sites, which are typically located next to a membrane that needs to move. This is possible because there is a kinetic barrier to new filament formation. ${ }^{[1]}$ New filaments are initiated extremely slowly under physiological conditions, but the process may be kick-started by proteins that form new actin "nuclei" that catalyse polymerization. Control of nucleating proteins is thus fundamental for cell movement.

M. B. Sweatman

School of Engineering

University of Edinburgh

King's Buildings, Mayfield Road, Edinburgh EH9 3FB, Scotland, UK

E-mail: martin.sweatman@ed.ac.uk

R. Insall

CR-UK Beatson Institute

Switchback Road, Bearsden, G61 1BD, Scotland, UK

R. Insall

Institute of Cancer Sciences

University of Glasgow

Garscube Estate, Switchback Road, Bearsden G61 1QH, Scotland, UK

The ORCID identification number(s) for the author(s) of this article can be found under https://doi.org/10.1002/adts.201800203

DOI: 10.1002/adts.201800203 membrane for several minutes or tens of minutes, after which their curvature increases, they bud off, and are taken into the cell. WASP (or its similar relative, N-WASP) almost universally appears on the pits, but very briefly, just before they are endocytozed. ${ }^{[4]}$ Most explanations for this process involve recruitment of the WASP proteins by direct binding to WASP recruiters on the coated pit. However, the details, including the precise recruiting agents and the mechanisms controlling their activity, have remained opaque.

All WASP family members include a polyproline domain, a long stretch containing multiple repeats of sequences such as XPPPPY, where P denotes a proline sidechain. Their actinnucleating activity is regulated by proteins such as Nck, which contain multiple copies of an $\mathrm{SH} 3$ domain that binds efficiently to single polyproline repeats. Nck itself, for example, contains three SH3 domains. There is thus a possibility of crosslinking large complexes of WASP family proteins and Nck. ${ }^{[5]}$ However, it is not known how these WASP-Nck aggregates form the discrete, size-limited structures on which their function depends. More specifically, as these aggregates do not exhibit bounding membranes or any other form of confinement, one might expect any WASP-Nck domain that nucleates to grow across the entire cell, limited only by the availability of WASP or Nck. But this is not observed. Neither the dynamic behavior of WASP, nor its restricted localization within membrane pits, is understood.

In fact, our ignorance of the basic physics of WASP-Nck domains exposes a more general lack of understanding of the biophysics of membraneless organelles, that is, self-limited protein structures within cells. ${ }^{[6]}$ WASP-Nck clusters are just one example of such structures, but many more are known, each 
with important cellular functions and specific structural and dynamic properties.

Typically, the physics of such membraneless organelles is explained in terms of liquid-liquid phase separation, ${ }^{[7]}$ where the liquid-like protein domains are thought to phase separate from the cytoplasm (the aqueous solution that fills cells). But this mechanism, by itself, is inadequate to explain their selflimiting structure or specific dynamic properties. The reason is that liquid-liquid phase separation normally produces coexisting bulk liquid phases rather than self-limiting structures, or clusters. Therefore, other presently unknown mechanisms must be in play that stabilize or limit aggregation to relatively small clusters of proteins.

Here, we propose that a simple model, the SALR model interaction, is capable of describing the observed physics of WASP clusters. The SALR (short-range attraction, long-range repulsion) model is already known to be capable of forming equilibrium, self-limited liquid-like clusters under specific conditions. ${ }^{[8]}$ Our aim is to test this idea through molecular-scale simulations of the WASP-Nck system. Detailed atomistic or molecular models of the interactions between WASP and Nck proteins are not needed, that is, chemical accuracy is unimportant for this study of basic physics. Instead, we will explore the behavior of a simple "coarsegrained" SALR model, where each SALR particle represents an entire protein interacting with other proteins in solution.

\section{The SALR Model}

Interest in the SALR model potential has grown steadily over at least the last decade through its application to a wide variety of important fluids, such aqueous dispersions of biological macromolecules (including proteins), colloids, and nanoparticles. ${ }^{[9]}$ Normally, it is used to represent the "effective" interaction between these types of solute in solution, in theories and simulations where the solvent is not modeled explicitly. That is, the presence of the solvent is taken into account, approximately, through definition of the SALR interaction. In technical language, the solvent is "integrated out".

Normally, the SALR pair potential is added to a particle core, which prevents particle overlaps;

$\varphi(r)=\varphi_{\text {core }}(r)+\varphi_{\text {SALR }}(r)$

where $r$ is the distance between a pair of particles. In this work, the core is modeled as a hard sphere with diameter $d$, and a 2Yukawa potential is used to model the SALR contribution

$$
\begin{aligned}
& \beta \varphi(x) \\
& =\beta \varphi_{H S}(x)+\beta \varphi_{2 Y}(x) \\
& =\left\{\begin{aligned}
\infty & ; \quad x<1 \\
-\frac{A_{a} \exp \left(-z_{a}(x-1)\right)}{x}+\frac{A_{r} \exp \left(-z_{r}(x-1)\right)}{x} ; & x \geq 1
\end{aligned}\right.
\end{aligned}
$$

where $x=r / d$ and $\beta=1 / k_{B} T$, with $k_{B}$ as Boltzmann's constant and $T$ the temperature. The SALR parameters $A_{a}, A_{r}, z_{a}$, and $z_{r}$ (all dimensionless and positive) define the magnitude and range of interactions beyond the core. In this work, we set $z_{a}=2$, $\mathrm{z}_{\mathrm{r}}=1.0$, and eliminate units by comparing energies to $k_{B} T$ and lengths to $d$.

The SALR model potential describes solutions where shortrange attractive (SA) interactions compete with long-range repulsive (LR) interactions. It is this competition, when suitably balanced, that leads to the self-limited aggregation, or giant equilibrium clusters, observed. The short-range attractive contribution models a wide range of common aggregation mechanisms, from direct van der Waals interactions to depletion and solvophobic effects. In the context of WASP-Nck interactions, the shortrange attraction accounts for a number of specific forces, including in particular SH3 domain-polyproline interactions. The long-range repulsions, on the other hand, normally represent a screened-coulomb interaction between like-charged solutes in a charge-neutralizing solution. Therefore, the SALR model is suitable for describing many kinds of solute that become charged, for example, through proton exchange, in solution. Given this condition applies to many biological macromolecules, including practically all proteins, then the SALR model should be suitable for describing the formation and phase behavior of self-limiting protein aggregates that form in the cytoplasm within biological cells.

Earlier simulation and theoretical work has revealed these SALR mesophases possess characteristics that appear to be widespread in cell biology. For example, Giant SALR clusters occur at thermodynamic equilibrium when the overall system concentration is sufficiently high, ${ }^{[19]}$ above a "critical cluster concentration" (CCC). The giant clusters formed tend to be relatively monodisperse in size and can appear like tiny liquid-like droplets undergoing Brownian motion. A first order phase transition is also apparent for a narrow range of interaction parameters between dispersed and condensed cluster phases. ${ }^{[11]}$

Furthermore, it has been shown recently that each giant SALR cluster acts as a nucleation center for the production of further clusters in systems where the SALR particle concentration is slowly increased. ${ }^{[17]} \mathrm{New}$ clusters are formed through a kind of fission, or "cluster reproduction event", that resembles the division of cells through mitosis. This led to the suggestion that SALR clusters might be capable of chemical evolution and therefore might be important in the origin of life. Moreover, they might also be capable of reproducing cellular structures like pseudopods that grow by fission ${ }^{[13]}$ rather than by being repeatedly created de novo.

Giant SALR clusters could therefore be a good model of membraneless intracellular structures, in particular, ones involving WASP family members whose discreteness has not yet been explained. However, the behavior of giant SALR clusters near membrane interfaces, which is central to the behavior of WASP-Nck clusters, has not yet been studied. Therefore, in the next section, we describe a simulation methodology able to illuminate the basic physics of giant SALR clusters at membrane surfaces.

\section{Monte Carlo Simulation of Giant SALR Cluster Adsorption at a Membrane Surface}

As this work is focused mainly on the equilibrium adsorption of SALR clusters at an interface, analogous to WASP and Nck proteins adsorbed on the intracellular surface of a membrane, 
an equilibrium simulation method with a fixed chemical potential, such as grand canonical Monte Carlo is preferred. These methods have the advantage that specially-designed Monte Carlo moves can accelerate sampling. This simulation protocol is described below.

All simulations consist of a rectangular simulation box of side length $80 \times 40 \times 40$, with periodic boundaries in each direction, and a long-ranged cutoff between all particles of 20. Each simulation box contains at most four species of particle. The membrane is modeled by a fixed number (1600) of hard spheres $\left(A_{a}=A_{r}=0\right.$ in Equation (2)) trapped within a harmonic external potential (with spring constant $k=10$ in reduced units) such that they form a compact layer across the central plane of the simulation box. Nck protein is modeled by an SALR particle at a fixed chemical potential. WASP protein, on the other hand, is modeled in terms of an SALR particle that can take two forms linked via a reversible reaction. Experiments have established that WASP family proteins typically exist initially in the cytosol (the aqueous phase within cells outside of membraneless organelles) as "closed," autoinhibited forms which diffuse relatively freely and do not affect actin. ${ }^{[14]}$ Following activation, they adopt an "open" conformation in which components such as the polyproline domains are accessible to protein-protein interactions.

In our model, the "closed" and "open" WASP forms have different sets of SALR interaction parameters. The closed form is assigned a chemical potential while the open form can be created or destroyed via the reaction mechanism, which is controlled by a reduced reaction (grand) free energy $U_{r}$. This is equivalent to a nonreacting system where both WASP forms have their own chemical potentials that differ by $\exp \left(U_{r}\right)$. Finally, the clathrin protein is modeled as an SALR particle with a strong cross-interaction with membrane and open WASP particles. In this way, the clathrincoated pits can "tether," or template, WASP-Nck clusters at specific positions.

The SALR parameters for both Nck and WASP proteins (both open and closed conformations) are chosen such that clusters do not form in the bulk fluid for any set of chemical potentials and reaction free energies used in these simulations. Trial bulk simulations initiated with a concentrated "ball" of Nck and WASP proteins are performed in the absence of any membrane to check that any SALR clusters that form initially are unstable, that is, the systems used are always below their respective bulk critical cluster concentration (CCC). Neither do any of the proteins form giant clusters at the interface by themselves under these conditions, that is, the mixture of open-WASP and Nck is required to form large clusters. For all simulations, $A_{r}=0.5$ except for interactions with the membrane, for which $A_{r}=0$. Table 1 lists all the corresponding values for $A_{a}$.

As our focus is on the chemical physics of these mixed clusters at an interface, chemical accuracy is not so important. We therefore choose all particles to have the same size. In reality, WASP proteins typically take on a spherical shape of around $7 \mathrm{~nm}$ in diameter, while Nck proteins are approximately $7 \mathrm{~nm} \times 3 \mathrm{~nm}$ cylinders. Although the relative abundances of WASP proteins is dominated by the closed form in the bulk cytosol, near a membrane surface, as in our simulations, their relative concentrations are thought to be quite similar. This difference in thought to be maintained by the action of other proteins embedded in the membrane, such as Cdc42. Therefore, for our near-membrane
Table 1. SALR interaction parameter $A_{a}$ for all simulations.

\begin{tabular}{lccccc}
\hline A_a & WASP (open) & WASP (closed) & Nck & Clathrin & Membrane \\
\hline WASP (open) & 1.5 & 1.5 & 2.0 & 2.0 & Varies \\
WASP (closed) & - & 1.5 & 1.5 & - & Varies \\
Nck & - & - & 1.5 & 1.5 & Varies \\
Clathrin & - & - & - & 3.0 & 1.5 \\
Membrane & - & - & - & - & 0
\end{tabular}

The core of each particle is identical; a hard sphere of unit diameter. A range of values are used for the cross interaction between protein and membrane particles.

simulations, we choose initially to set the reaction free energy between the two forms to be zero, reflecting their balanced populations in this region.

The length of a simulation is measured in terms of cycles, where a cycle consists of an attempt to move each particle plus an attempt to insert a particle, an attempt to delete a particle, and an attempt to swap the positions of a pair of nonidentical particles chosen randomly. Insertion and deletion attempts are only performed for those particle types with an associated chemical potential. For simulations involving reaction equilibria between WASP particle types, one reaction move is also attempted per cycle. Displacement move attempts can be short or long, chosen randomly, where the displacement move has a maximum length of 0.35 and 10, respectively. A reaction move attempts to transform a WASP particle chosen at random into the other form. The usual Metropolis criteria based on the Boltzmann factor are used to accept or reject all moves.

Three sets of simulations are performed that seek to understand how the equilibrium adsorption behavior of clusters depends on i) the cross-interaction strength between open WASP and membrane particles, ii) WASP reaction free energy, and iii) the presence of clathrin in the membrane. For all simulations, the system is initiated with a high concentration of WASP and clathrin (if included in the simulation) at the membrane interface so that any nucleation free energy barrier for cluster formation is avoided.

Results are reported in terms of final simulation frame snapshots and equilibrium density profiles of protein particles within clusters normal to the plane of the membrane. A protein particle is considered part of a cluster if it is within $2^{0.5}$ of any other protein particle. Adsorption can occur on both sides of the membrane, and therefore density profiles are reported as an average. Equilibrium averages are calculated from the final 100000 cycles of a simulation, after checking the system energy has converged.

\section{Results}

First, we consider the case when the reaction free energy difference between open and closed WASP forms is zero. For this system, three simulations were performed with different proteinmembrane cross-interaction strengths; $A_{a}=0.25,0.375$, and 0.5 . Figure 1a shows final snapshots from these simulations, while Figure $1 \mathrm{~b}$ shows the corresponding density profiles of clustered particles. 

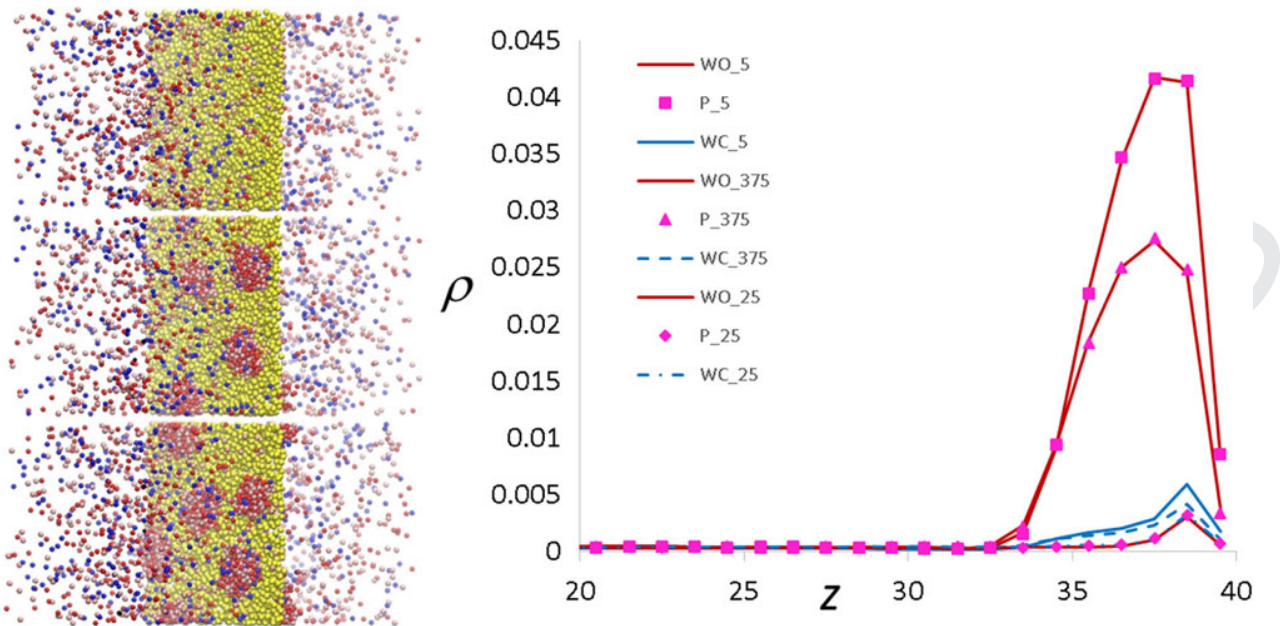

Figure 1. Left: snapshots from the end of simulations for the WASP-membrane system with different protein-membrane interactions strengths $A_{a}$; top $=0.25$, middle $=0.375$, bottom $=0.5$. Red and blue spheres are open and closed WASP proteins, respectively, pink spheres are Nck proteins, and yellow spheres are membrane particles. The simulation cell has been rotated to provide a good view. Right: corresponding density $(\rho)$ profiles of particles in clusters. The membrane (not plotted) is located at $z=40$, where $z$ measures the distance along the simulation cell normal to the membrane. WO, WC, and P represent the open and closed WASP forms, and Nck protein, respectively. Statistical errors are generally less than symbol sizes.
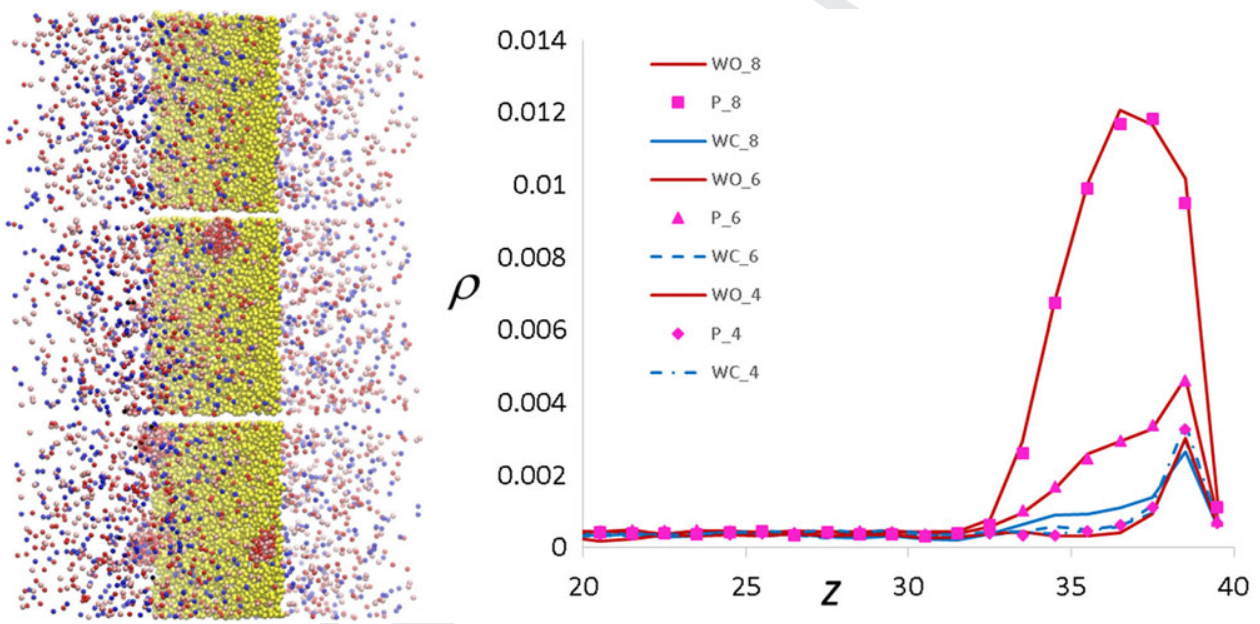

Figure 2. As for Figure 1, except the interaction with the membrane is held fixed (at $A_{a}=0.25$ ) while the reaction free energy, $U_{r}$, is varied. Clusters are observed when the reaction free energy is above 0.04 , that is, when it is 0.06 and 0.08 here.

Clearly, when the protein cross-interaction with the membrane is weak $\left(A_{a}=0.25\right)$, giant SALR clusters are unstable. But for higher interaction strengths, stable clusters form on the membrane surface, even though they are unstable in the bulk. Essentially, adsorption of protein at the membrane surface can drive it above the cluster critical concentration, at least locally, such that clusters are stable at the interface. The clusters are formed primarily of the open form of WASP and Nck.

For the next set of simulations, the cross-interaction strength of all proteins with the membrane is fixed at $A_{a}=0.25$, which, from the preceding simulations, corresponds to an absence of clusters at the interface for zero activated-closed WASP reaction free energy. Therefore, to obtain clustering at the membrane interface, the reaction free energy is increased slightly above zero, meaning that the activated (open) form of WASP is slightly favored over the autoinhibited (closed) one, in steps of 0.02 .
Figure 2 shows the corresponding simulation snapshots and density profiles. It is evident that for this specific membraneprotein cross interaction strength clusters at the membrane interface become stable above a reaction free energy difference of 0.04 , that is, when the open WASP form is slightly favored. Once again, cluster formation occurs when there is a sufficient concentration of protein at the membrane surface, despite clusters being unstable in the bulk. It is interesting to note that the cluster size is relatively insensitive to either the interaction strength with the surface or the reaction free energy difference. This is expected, as giant SALR cluster size has previously been found to depend mainly on the SALR pair potential itself (i.e., the interaction between WASP and Nck rather than either's interaction with the membrane).

In the final simulations, clathrin protein is introduced into the simulation cell and autoinhibited "closed" WASP is absent. To 
(a)

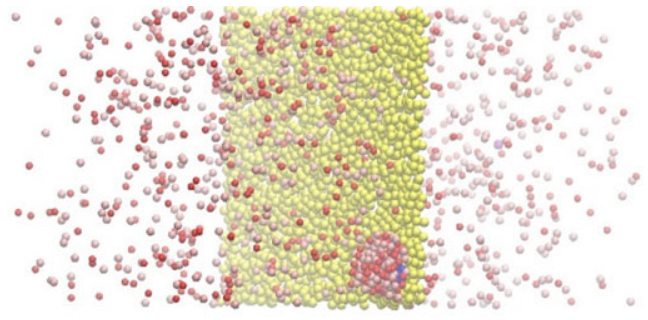

(b)
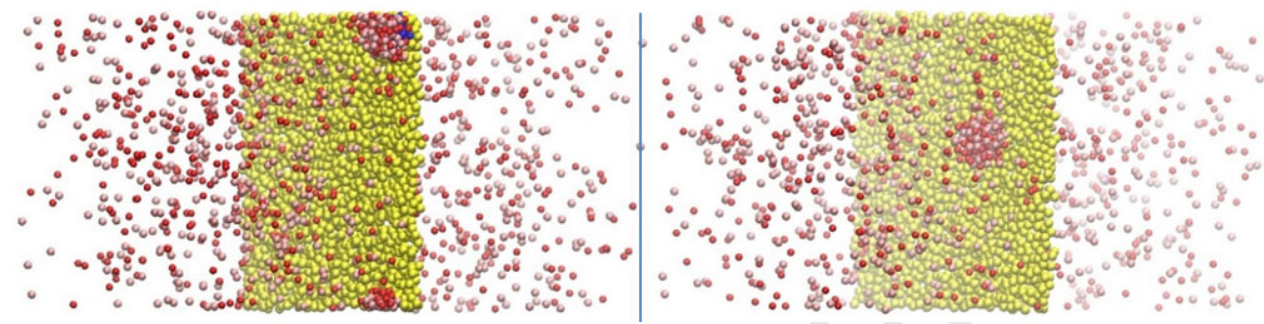

Figure 3. a) As for Figure 1, except blue spheres now represent clathrin (and there is no autoinhibited WASP). Clathrin forms a cluster at the membrane surface which is obscured by the WASP-Nck SALR cluster formed on top of it. In this case, in the absence of clathrin, WASP-Nck clusters are unstable everywhere. b) As for Figure 3a, except the protein-membrane interaction strength $(0.26)$ is chosen such that WASP-Nck clusters are unstable in the bulk, but marginally stable at the membrane surface. Left: simulation with clathrin. Right: simulation without clathrin.

maintain open WASP within the simulations, it is now assigned a chemical potential and does not undergo any chemical reaction to the autoinhibited form. The clathrin-clathrin pair interaction is chosen such that it forms clusters within the membrane interface, mimicking the formation of clathrin coated pits during pinocytosis. In the first such simulation, the interaction strength of WASP and Nck particles with the membrane is $A_{a}=0.25$ and the reaction free energy difference is $U_{r}=0.0$ (i.e., open WASP has the same chemical potential as the closed form previously), such that WASP-Nck clusters are unstable both in the bulk and at the interface. In the second simulation, the interaction strength of WASP and Nck particles with the membrane is instead set to 0.26 , such that WASP-Nck clusters are only marginally stable at the interface. Comparison is made with an identical simulation performed in the absence of clathrin.

Figures 3a clearly shows that even when giant SALR clusters are unstable in the bulk and at the interface, they can be stabilized by the presence of an attractive site at the membrane surface, that is, by clathrin. Interestingly, Figure $3 \mathrm{~b}$ shows that when clusters are stable at the membrane surface (but not in the bulk), they will tend to form around a clathrin cluster if one exists. This is not surprising from a chemical physics perspective-we expect this adsorption state to occur because it has a lower free energy than the situation where clathrin and WASP-Nck clusters are separated. However, it is potentially very important from a biological perspective, as discussed next.

\section{Discussion}

It is typical in biology to discuss structures as being "induced"that is to say, under appropriate conditions, the presence of some upstream information causes the structure to form, and delimits both where it occurs and at what time. In the case of WASP, the founding member of the broad WASP family, the structures are small puncta next to the membrane. In adherent cells, puncta are typically observed on the base where the cells adhere; it is unclear whether they are concentrated there, or just easier to detect. ${ }^{[15\rfloor}$ The WASP puncta are usually assembled at the sites of clathrin-coated pits, which are part of the cell's endocytosis machinery. In mammalian cells, essentially all clathrin pits colocalize briefly with puncta of the ubiquitous homologue N-WASP..$^{[4]}$ The N-WASP assembles just before the clathrin pits move from the surface toward the interior of the cell. It is thus typical in the biological literature to state that the clathrin pits "induce" or "cause" the N-WASP puncta. This implies that clathrin pits are both necessary (implying that without clathrin, N-WASP is not recruited) and sufficient (implying that the coated pits, composing clathrin and other proteins, strictly specify both the time at which N-WASP puncta may form, and the places they arise). However, these issues are rarely tested experimentally. We know no work demonstrating that clathrin is essential for N-WASP puncta, and the pioneering work of Drubin has shown that causation during coated pit evolution is far more complex than others have presumed. ${ }^{[16]}$

Here, we suggest that a different relationship between WASP and clathrin coated pits might be in play. We propose that WASP puncta form as SALR clusters together with Nck stochastically rather than under the control of an upstream "activator." This possibility is suggested explicitly by the results in Figure 3 . If this is true, then we suggest clathrin biases the position of SALR clusters, making them more likely to form next to clathrin-coated pits. Nevertheless, they are also able to form in the absence of clathrincoated pits.

Overall, the SALR model is very successful at mimicking the basic physics of WASP-Nck membraneless organelles. It provides a clear mechanism for the equilibrium formation of protein aggregates with specific sizes and lifetimes, which agrees accurately with other groups that have observed physiological aggregation of proteins that interact strongly. The more commonly 
invoked mechanism of liquid-liquid phase separation cannot, by itself, explain how size-limited protein aggregates form with such fine control. As polyvalent motifs and aggregation to form sizelimited clusters are common in biology, we suggest this model can more generally be used as a way to describe the formation and control of membraneless organelles.

\section{Conflict of Interest}

The authors declare no conflict of interest.

\section{Keywords}

$\mathrm{xxx}$

Received: December 20, 2018 Revised: February 9, 2019

Published online:

[1] J. A. Cooper, E. L. Buhle, S. B. Walker, T. Y. Tsong, T. D. Pollard, Biochemistry 1983, 22, 2193.

[2] S. B. Padrick, M. K. Rosen, Annu. Rev. Biochem. 2010, 79, 707.
[3] D. M. Veltman, R. H. Insall, Mol. Biol. Cell 2010, 21, 2880.

[4] C. J. Merrifield, B. Qualmann, M. M. Kesses, W. Almers, Eur. J. Cell Biol. 2004, 83, 13.

[5] P. Li, S. Banjade, H. C. Cheng, S. Kim, B. Chen, L. Guo, M. Llaguno, J. V. Hollingsworth, D. S. King, S. F. Banani, P. S. Russo, Q. X. Jang, B. T. Nixon, M. K. Rosen, Nature 2012, 483, 336.

[6] U. S. Eggert, Biochemistry 2018, 57, 2403.

[7] E. Gomes, J. Shorter, J. Biol. Chem. 2018, in press.

[8] M. B. Sweatman, R. Fartaria, L. Lue, J. Chem. Phys. 2014, 140, 124508.

[9] a) A. Stradner, H. Sedgwick, F. Cardinaux, W. C. K. Poon, S. U. Egelhaaf, P. Schurtenbuerger, Nature 2004, 432, 492; b) N. Meihac, N. Destainville, J. Phys. Chem. B 2011, 115, 7190; c) C. P. Royall, Soft Matter 2018, 14, 4020; d) M. K. Bera, B. F. Qiao, S. Seifert, B. P. BurtonPye, M. O. de la Cruz, M. R. Antonio, J. Phys. Chem. C 2016, 120, 1317; e) Z. Varga, J. Swan, Soft Matter 2016, 12, 7670; f) Y. Zhuang, P. Charbonneau, J. Phys. Chem. B 2016, 120, 7775.

[10] M. B. Sweatman, R. Fartaria, L. Lue, J. Chem. Phys. 2014, 140, 124508.

[11] M. B. Sweatman, L. Lue, J. Chem. Phys. 2016, 144, 171102.

[1Z] M. B. Sweatman, Mol. Phys. 2018, 116, 1945.

[13] N. Andrew, R. H. Insall, Nat. Cell Biol. 2007, 9, 193.

[14] A. S. Kim, L. T. Kakalis, N. Abdul-Manan, G. A. Liu, M. K. Rosen, Nature 2000, 404, 151.

[15] A. J. Davidson, C. Amto, P. A. Thomason, R. H. Insall, J. Cell Biol. 2018, 217, 701.

[16] J. Weinberg, D. G. Drubin, Trends Cell Biol. 2012, 22, 1. 


\section{ADVANCED THEORYAND SIMULATIONS}

Reprint Order Form 2019
Editorial Office:

Wiley-VCH Verlag

Boschstraße 12, 69469 Weinheim

Germany

Tel.: (+49) 3047031331

Fax: (+49) 6201606510

Email: advtheorysimul@wiley.com

\section{Short DOI: adts.}

Please send me and bill me for

no. of Reprints via $\square$ airmail (+ 25 Euro) $\square$ surface mail

Please send me and bill me for $a$

high-resolution PDF file (330 Euro).

My Email address:

Please note: It is not permitted to present the PDF file on the internet or on company homepages.

\section{Information regarding VAT}

Please note that from German sales tax point of view, the charge for Reprints, Issues or Posters is considered as "supply of goods" and therefore, in general, such delivery is a subject to German sales tax. However, this regulation has no impact on customers located outside of the European Union. Deliveries to customers outside the Community are automatically tax-exempt. Deliveries within the Community to institutional customers outside of Germany are exempted from the German tax (VAT) only if the customer provides the supplier with his/her VAT number. The VAT number (value added tax identification number) is a tax registration number used in the countries of the European Union to identify corporate entities doing business there. It starts with a country code (e.g. FR for France, GB for Great Britain) and follows by numbers.

VAT no.:

(Institutes / companies in EU countries only)

Purchase Order No.:
Delivery address / Invoice address:

Name of recipient, University, Institute, Street name and Street number, Postal Code, Country

\section{Date and Signature:}

Credit Card Payment (optional) -You will receive an invoice.

\section{VISA, MasterCard, AMERICAN EXPRESS}

Please use the Credit Card Token Generator located at the website below to create a token for secure payment. The token will be used instead of your credit card number.

\section{Credit Card Token Generator:}

https://www.wiley-vch.de/editorial production/index.php

Please transfer your token number to the space below.

Credit Card Token Number

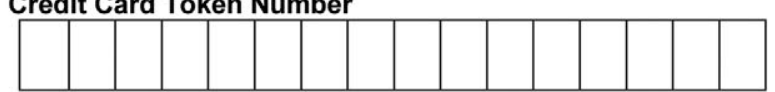

Price list for reprints (The prices include mailing and handling charges. All Wiley-VCH prices are exclusive of VAT)

\begin{tabular}{|c|c|c|c|c|c|c|}
\hline No. of pages & 50 copies & 100 copies & $\begin{array}{l}\text { Price (in El } \\
150 \text { copies }\end{array}$ & $\begin{array}{l}\text { for orders of } \\
200 \text { copies }\end{array}$ & 300 copies & 500 copies \\
\hline $\begin{array}{l}1-4 \\
5-8\end{array}$ & $\begin{array}{l}345 \\
490\end{array}$ & $\begin{array}{l}395 \\
573\end{array}$ & $\begin{array}{l}425 \\
608\end{array}$ & $\begin{array}{l}445 \\
636\end{array}$ & $\begin{array}{l}548 \\
784\end{array}$ & $\begin{array}{c}752 \\
1077\end{array}$ \\
\hline $9-12$ & 640 & 739 & 786 & 824 & 1016 & 1396 \\
\hline $13-16$ & 780 & 900 & 958 & 1004 & 1237 & 1701 \\
\hline
\end{tabular}

Wiley-VCH Verlag GmbH \& Co. KGaA; Location of the Company: Weinheim, Germany;

Trade Register: Mannheim, HRB 432833, Chairman of the Board: Mark Allin General Partner: John Wiley \& Sons GmbH, Location: Weinheim, Germany

Trade Register Mannheim, HRB 432296,

Managing Directors: Sabine Steinbach, Dr. Guido F. Herrmann 